1 Programa Saúde da Família (PSF) - Niterói (RJ), Brasil. marianajanssen@hotmail. com

2 Universidade Federal Fluminense (UFF), Instituto de Saúde Coletiva (ISC), Departamento de Epidemiologia e Bioestatística - Niterói (RJ), Brasil. sandracfonseca@yahoo. combr

3 Universidade Federal Fluminense (UFF), Instituto de Saúde Coletiva (ISC), Departamento de Epidemiologia e Bioestatística - Niterói (RJ), Brasil.

giselecalexandre@gmail.com

\section{Avaliação da dimensão estrutura no atendimento ao pré-natal na Estratégia Saúde da Família: potencialidades do PMAQ-AB}

\author{
Structure Evaluation of Prenatal Care in the Family Health Strategy: \\ $P M A Q-A B$ strengths
}

Mariana Janssen', Sandra Costa Fonsecaㄹ, Gisele Caldas Alexandre ${ }^{\mathbf{3}}$

RESUMO Esta pesquisa analisou a dimensão estrutura das unidades para a atenção pré-natal em Niterói, realizada pela Estratégia Saúde da Família (ESF). O banco de dados foi o da avaliação externa do $1^{\circ}$ ciclo do Programa Nacional de Melhoria do Acesso e Qualidade da Atenção Básica (PMAQ-AB) do Ministério da Saúde. Foram entrevistados 69 profissionais, em 27 unidades de saúde. Com base nos resultados, propõe-se melhorar as condições de acessibilidade, qualificar os profissionais, estimular a permanência e o vínculo, ampliar a capacitação das equipes quanto ao gerenciamento do território e dos protocolos de risco. O PMAQ-AB foi corroborado como uma ferramenta para avaliações em saúde.

PALAVRAS-CHAVE Cuidado pré-natal. Avaliação de serviços de saúde. Estrutura dos serviços. Estratégia Saúde da família.

ABSTRACT This research evaluated the structure dimension of primary care centers offering prenatal care in the Family Health Strategy (ESF) of Niterói. The database came from an external National Program for Access and Quality Improvement in Primary Care (PMAQ-AB) evaluation, conducted by the Federal Ministry of Health, between 2012 and 2013. Data came from 69 interviews with health professionals, spread throughout all 27 primary health units of the ESF. Based on results, we propose the improvement of accessibility in health units, professional training, binding between patients and the team, and increase the health team's knowledge of territory management and risk guidelines. We corroborated $P M A Q-A B$ as a tool for health evaluation.

KEYWORDS Prenatal care. Health services evaluation. Structure of services. Family Health Strategy. 


\section{Introdução}

A Atenção Básica (AB) configura-se como a porta de entrada prioritária para o Sistema Único de Saúde (SUS) e, entre suas atribuições, encontra-se a atenção pré-natal de baixo risco (BRASIL, 2013). Para a execução de um atendimento de qualidade no pré-natal, uma das dimensões necessárias é a estrutura.

Entre os estudos brasileiros sobre avaliação da qualidade da atenção pré-natal, poucos abordaram a dimensão estrutura e outras questões importantes como formação e capacitação dos recursos humanos e disponibilidade de imunobiológicos (XIMENES ET AL., 2008; ROCHA ET AL., 2012; OLIVEIRA ET AL., 2013; SILVA ET AL., 2013; OLIVEIRA 2014).

Em 2011, foi implantado, no Brasil, o Programa Nacional de Melhoria do Acesso e da Qualidade da Atenção Básica (PMAQAB) pelo Ministério da Saúde (BRASIL, 2011B). Os dados do PMAQ-AB abrangem todas as atividades prioritárias da $\mathrm{AB}$, sendo possível extrair um conjunto de informações sobre a dimensão estrutura das unidades. Os dados possibilitam também a realização de avaliações comparativas entre equipes, municípios, estados ou macrorregiões. Em Niterói, 90\% das Equipes de Saúde da Família (EqSF) aderiram ao PMAQ-AB em 2011. Essas equipes foram submetidas à primeira avaliação externa entre 2012 e 2013, simultaneamente a todos os outros municípios do País (Núcleo Gestor de Informações da Saúde da Família de Niterói, consultado em 15 de junho de 2015).

Não foram encontrados estudos publicados que avaliassem a qualidade da atenção pré-natal utilizando os dados do PMAQ-AB. Este estudo se propôs a avaliar a dimensão estrutura como componente da qualidade dos serviços que oferecem pré-natal, utilizando as informações disponibilizadas, entre os anos de 2012 a 2013, sobre as unidades de saúde da família de Niterói.

\section{Material e métodos}

Trata-se de um estudo descritivo transversal, realizado no município de Niterói, estado do Rio de Janeiro, região Sudeste, Brasil, que apresenta o maior índice de desenvolvimento humano municipal (IDHM=0,837) do estado (IBGE, 2016) e está na sétima posição no ranking nacional. A população residente em 2010 era de 487.562 pessoas, sendo 261.724 mulheres. Destas, 115.560 tinham entre 15 e 45 anos de idade, segundo o censo de 2010 (IBGE, 2016). A cobertura da Estratégia Saúde da Família (ESF) entre 2012 e 2013 em Niterói era de aproximadamente $38 \%$ da população total (Núcleo Gestor de Informações da Saúde da Família de Niterói, consultado em 15 de junho de 2015).

A avaliação externa do PMAQ-AB foi realizada entre 2012 e 2013 e coordenada pela Fundação Oswaldo Cruz (Fiocruz). Das EqSF do município, 90\% aderiram ao Programa, o que corresponde a 27 de 32 unidades em funcionamento no ano da entrevista. As outras unidades não participaram da avaliação por ainda não terem sido inauguradas no momento da adesão das equipes ao Programa. Todas as unidades de saúde estão localizadas em área urbana.

Foi utilizado o banco de dados do PMAQ-AB referente ao período de 2012 a 2013, que se divide em três módulos. Foram selecionados para este trabalho os indicadores pertencentes aos módulos I e II, que permitissem a avaliação da dimensão estrutura para uma adequada atenção pré-natal (BRASIL, 2011A). Os dados do PMAQ-AB escolhidos foram agrupadas em cinco dimensões: Recursos Humanos (quantidade e formação das equipes por unidade de saúde, qualificação do profissional respondente e apoio matricial); Planta Física da Unidade (aspectos de acessibilidade, identificação do Território de abrangência); Equipamentos e Insumos para atendimento do pré-natal; Normas 
(turnos e horários de atendimento, rotinas especificadas, agenda de atendimento); Rede de Serviços (sistema de referência e regulação de vagas). Realizou-se uma análise descritiva das variáveis estudadas, utilizando o programa estatístico SPSS , versão 21.

$\mathrm{O}$ projeto de pesquisa original foi analisado e aprovado pelo Comitê de Ética em Pesquisa da Escola Nacional de Saúde Pública Sergio Arouca/Fiocruz, instituição coordenadora do PMAQ-AB, mediante protocolo $n^{\circ} 32.012 / 2012$. Os dados do primeiro ciclo já estão publicamente disponibilizados para fins de pesquisa. Não há qualquer procedimento de risco envolvendo os participantes. Os dados não contêm identificação de pacientes e profissionais. As unidades também tiveram suas identificações protegidas, portanto há garantia de sigilo e confidencialidade.

\section{Resultados}

Dos profissionais entrevistados, 39 eram médicos, 27 eram enfermeiros e 3 eram dentistas, sendo que $24(34,8 \%)$ atuam há menos de 1 ano na equipe de saúde local. Saúde da família ou medicina de família, pediatria e tocoginecologia compuseram $57 \%$ das especialidades relatadas pelos médicos entrevistados. Dez profissionais médicos $(25,6 \%)$ não possuíam formação complementar à graduação, enquanto todos os 27 enfermeiros tinham formação complementar, sendo $74 \%$ em saúde da família, saúde pública ou saúde coletiva. Nenhum dos dentistas possuía formação complementar à graduação. Apenas nove unidades de saúde ofereciam atendimento de saúde bucal.

Com relação à população de referência, a média de pessoas cadastradas por equipe de saúde foi de 1.863 indivíduos. Dos 69 profissionais entrevistados, 64 (94\%) referiram receber apoio institucional permanente de uma pessoa ou da equipe da Secretaria Municipal de Saúde com o objetivo de discutir, de forma conjunta, o processo de trabalho; 61 recebiam apoio presencial e 60, também por telefone. Dos que recebiam apoio matricial, 59 recebiam de 7 a 9 apoiadores na unidade de saúde. A quase totalidade (99\%) dos entrevistados referiu que a gestão disponibilizava informações úteis para a análise de situação de saúde da população, e todos relataram a realização de ações de educação permanente para profissionais da $\mathrm{AB}$ no município.

A acessibilidade das unidades de saúde pesquisadas foi considerada precária, uma vez que em apenas $37 \%$ havia rampa de acesso; em 15\%, a presença de corrimão, portas de entrada e internas adaptadas para cadeira de rodas; e em $7 \%$, corredores adaptados para cadeira de rodas. Somente quatro unidades tinham cadeira de rodas disponível (gráfico 1). 
Gráfico 1. Distribuição da frequência absoluta das variáveis de acessibilidade em unidades de Estratégia Saúde da Família, Niterói (RJ) - PMAQ-AB, 2012-2013 (n=27)

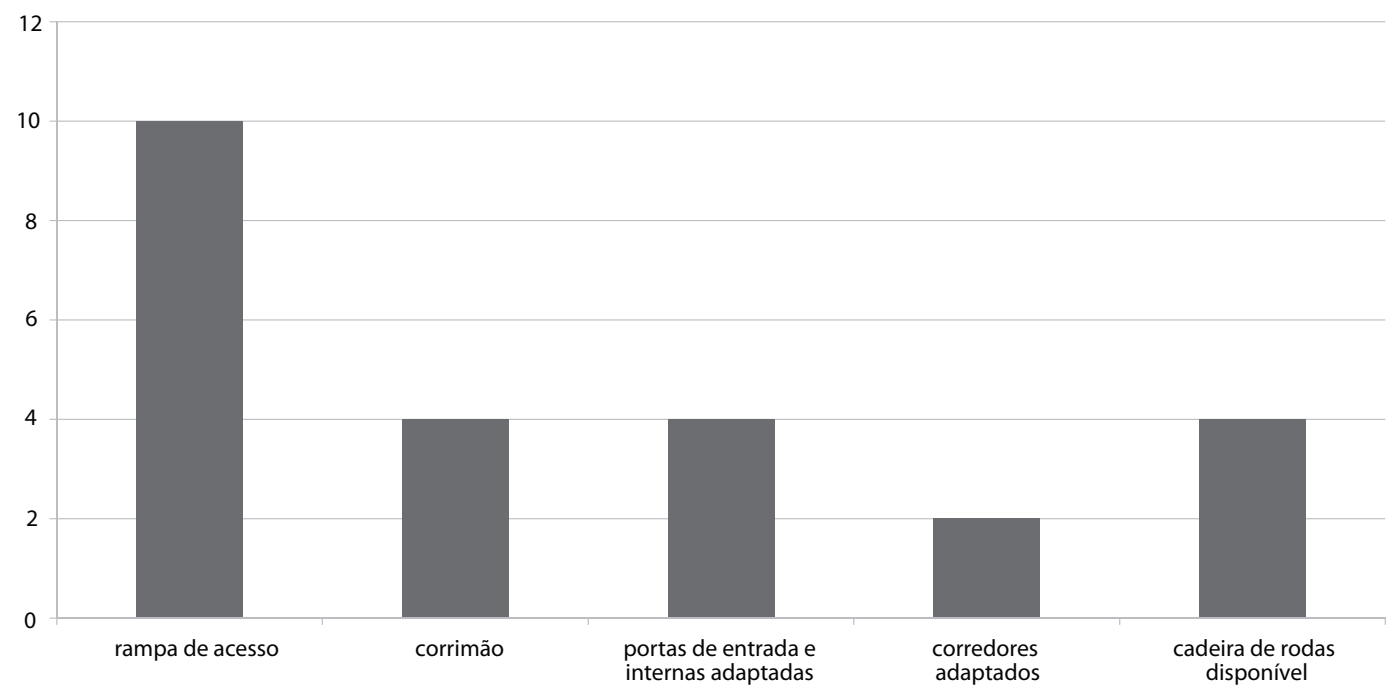

Com relação à estrutura predial, os ambientes mais deficientes nas unidades foram o consultório odontológico, a sala para estoque de medicamentos, a sala para acolhimento e o banheiro para usuárias (gráfico 2).

Gráfico 2. Distribuição de frequência absoluta dos itens de estrutura predial em unidades de Estratégia Saúde da Família, Niterói (RJ) - PMAQ-AB, 2012-2013 ( $n=27)$

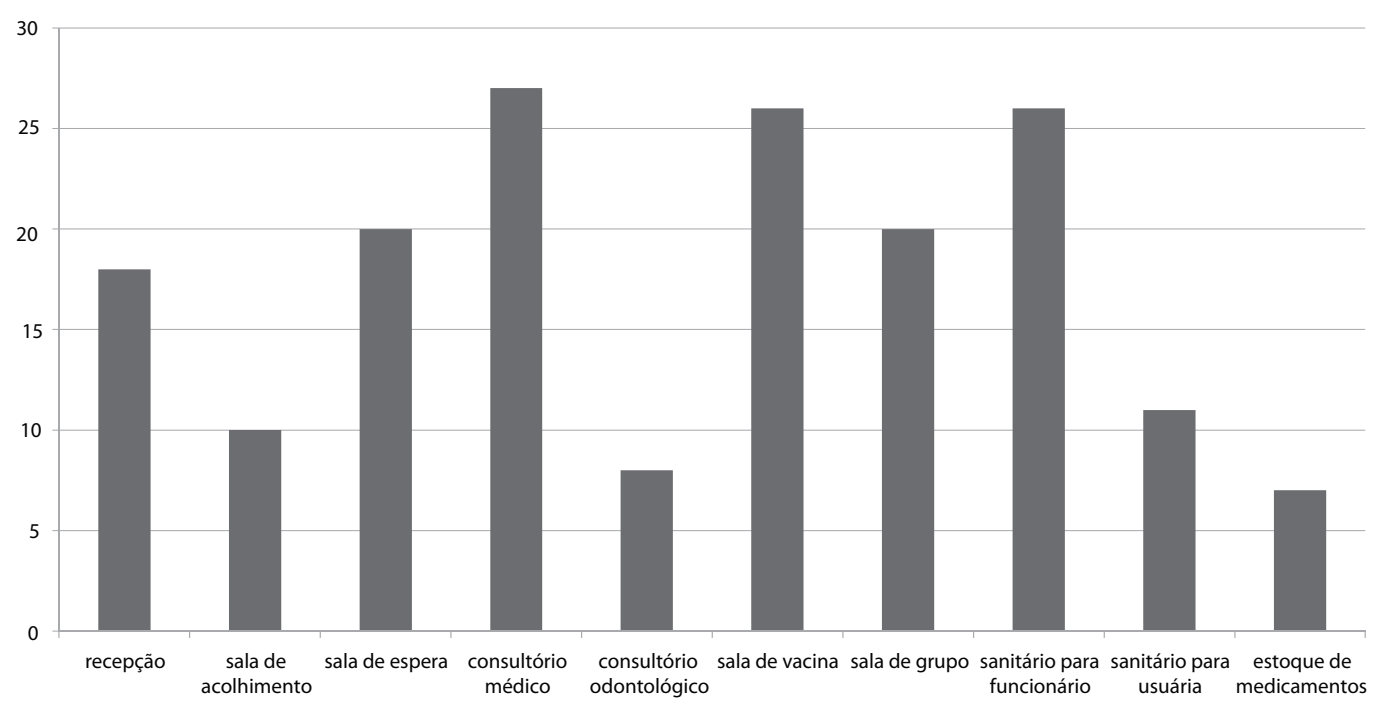

Quanto à ambiência, os itens mais precáparedes e pisos não laváveis (gráfico 3). rios foram a acústica e a cobertura de teto, 
Gráfico 3. Distribuição de frequência absoluta dos itens de ambiência em unidades de Estratégia Saúde da Família, Niterói (RJ) - PMAQ-AB, 2012-2013 ( $n=27)$

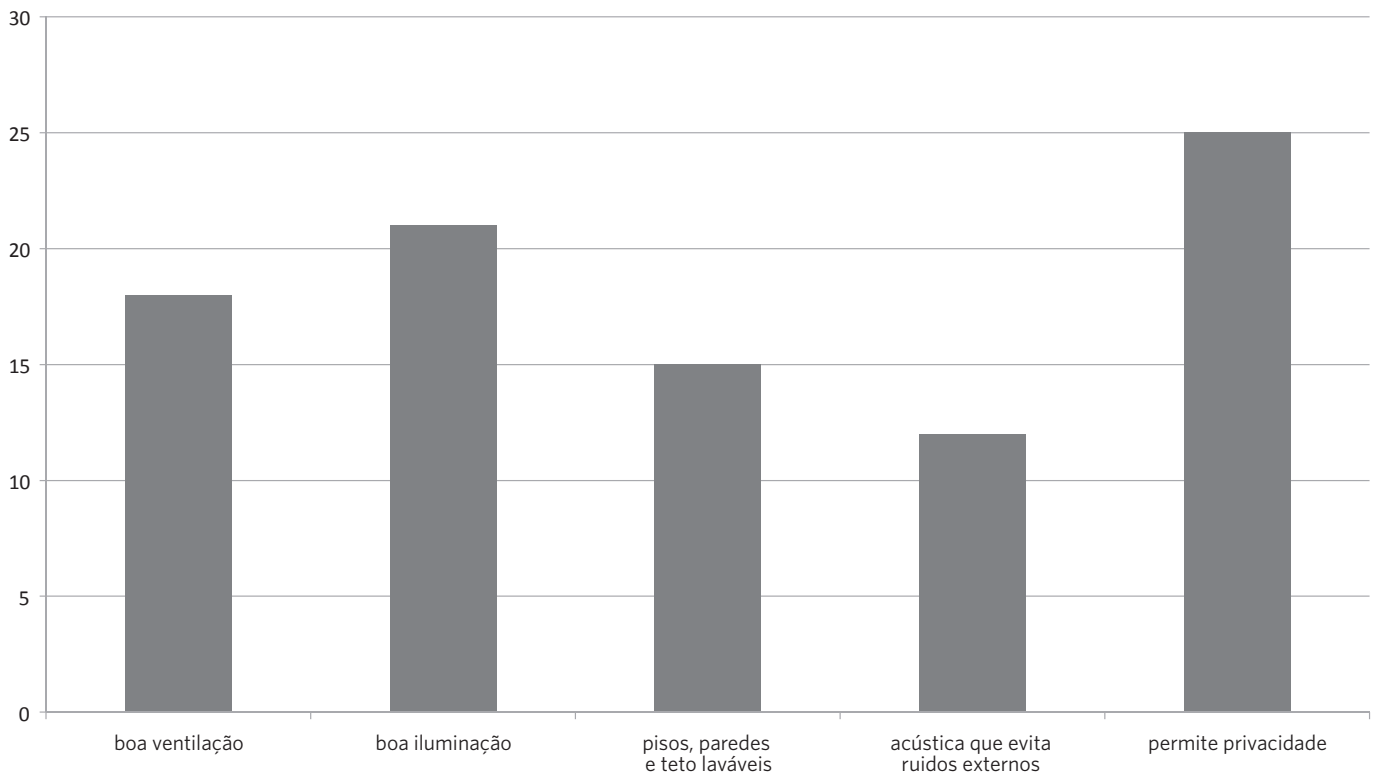

$\mathrm{Na}$ avaliação sobre equipamentos e insumos de tecnologia da informação, 25 unidades $(92,6 \%)$ possuíam computadores em condições de uso; 15 (55,6\%) tinham acesso à internet e $3(11,1 \%)$ possuíam Telessaúde. Nenhuma unidade possuía consultório com computador interligado à internet.

Quanto aos equipamentos gerais e materiais, todas as unidades investigadas possuíam aparelho de pressão para adulto, estetoscópio para adulto, glicosímetro, foco de luz para exame ginecológico, mesa para exame ginecológico com perneira, sonar ou estetoscópio de Pinard, caixas térmicas para vacinas, fita métrica e balança antropométrica. Uma unidade não dispunha de mesa para exame clínico, mas dispunha de mesa para exame ginecológico; duas não tinham geladeira para vacinas, e quatro não tinham equipamento de proteção individual (EPI) sempre disponíveis (luvas, máscaras, óculos, aventais). Apenas sete (26\%) unidades dispunham de geladeira exclusiva para farmácia.

A ficha de atendimento usada no pré-natal e as fichas de encaminhamento de usuárias para os demais pontos de atenção estavam presentes e em quantidade suficiente em todas as unidades. A ficha de requisição e de resultado de citopatológico do colo de útero e cartão de vacinação foram encontrados em 92,6\%; e a caderneta de gestante, em $81,5 \%$ das unidades.

Em 26 (96,3\%) unidades, estavam disponíveis as vacinas dupla tipo adulto-DT e hepatite B. A vacina de influenza sazonal estava presente em 18 (66,7\%) unidades.

Em todas as unidades, foram referidos como presentes e suficientes os seguintes itens: espéculo descartável, escovinha endocervical, lâmina de vidro com lado fosco, frasco plástico com tampa para lâmina, seringas e agulhas descartáveis; em 26 (96,3\%), a espátula de Ayres e fitas reagentes para glicemia capilar e gaze em 25 (92,6\%).

Os suplementos vitamínicos e antianêmicos, os analgésicos e o antiemético metoclorpramida estavam presentes e em quantidade suficiente em 25 (93\%) unidades, enquanto Insulina regular e NPH e os antibióticos, em 
24 unidades (89\%). O anti-hipertensivo cloridrato de hidralazina era suficiente em 15 unidades de saúde (55,55\%). Miconazol foi a única pomada ginecológica encontrada na verificação e estava presente e suficiente na maioria das unidades (gráfico 4).

Gráfico 4. Distribuição de frequência absoluta das variáveis de presença e suficiência de componentes da farmácia básica em unidades de Estratégia Saúde da Família, Niterói (RJ) - PMAQ-AB, 2012-2013 (n=27)

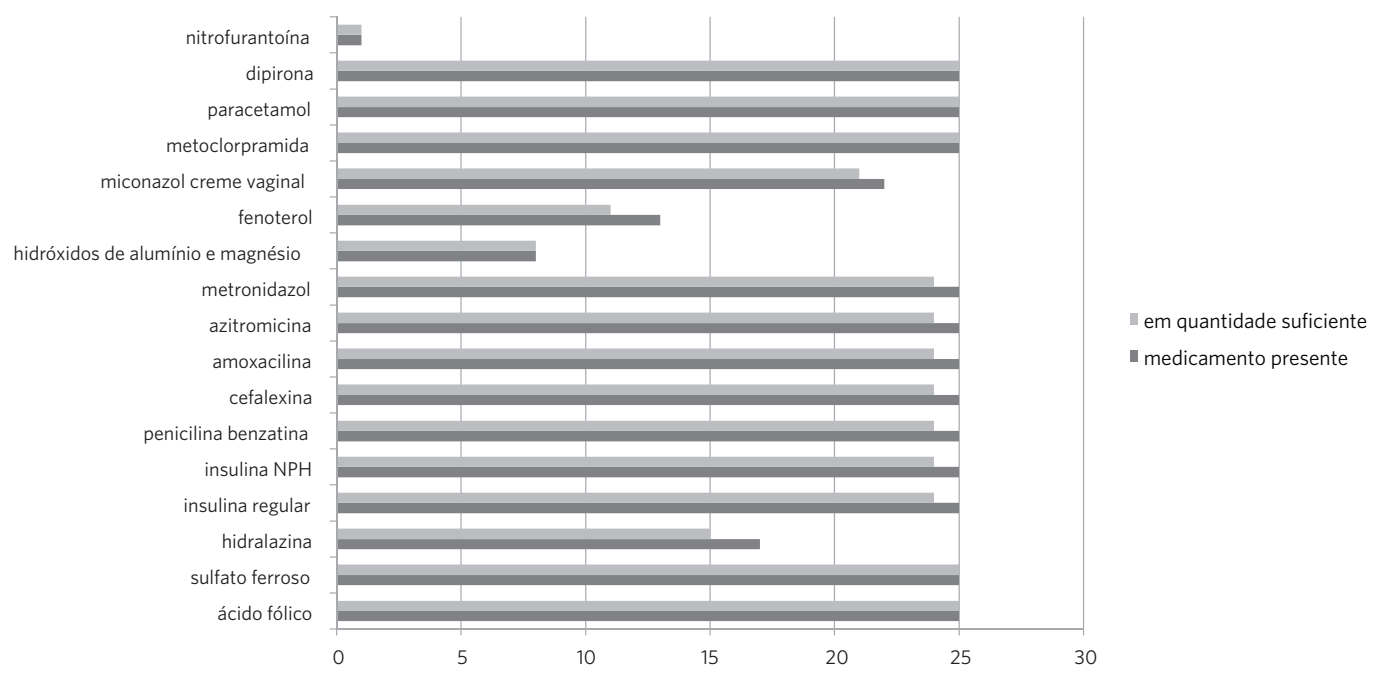

Todas as unidades funcionavam em dois turnos, manhã e tarde, das $8 \mathrm{~h}$ às $17 \mathrm{~h}$. No entanto, apenas três (11\%) funcionavam no horário de almoço. Todas tinham programação de oferta de consultas para atendimento pré-natal. Em todas elas havia coleta de exames de sangue, urina e citopatológico de colo de útero. Dispensação de medicamentos, realização de curativos e de vacinas estavam disponibilizadas aos usuários em $92,6 \%$ delas. Acolhimento e recepção aos usuários estavam disponíveis em $85,2 \%$ das unidades. Apenas 22 entrevistados (31,9\%) relataram que a unidade possuía protocolo com definição de diretrizes terapêuticas para acolhimento à captação precoce de gestantes ou à demanda espontânea de intercorrências na gestação. Sessenta e quatro entrevistados (92,7\%) referiram programar consultas e ações para gestantes em sua agenda, e 50
(72,5\%) utilizar protocolos para orientação e priorização dos casos que precisavam de encaminhamento para a atenção pré-natal de alto risco.

Todos os entrevistados afirmaram que a equipe realizava visitas domiciliares, mas apenas $22(32 \%)$ referiram protocolos de definição dos casos prioritários para a visita. A agenda organizada para realização de atividades educativas com grupos para gestantes foi referida por 64 equipes; pelo menos uma equipe por unidade de saúde afirmou essa organização. Todas as equipes pesquisadas disponibilizavam de central de regulação de vagas. Todavia, $13 \%$ referiram não ter definição de maternidade para as gestantes. Sessenta e cinco $(94,2 \%)$ dispunham de marcação de consultas especializadas, e 68 (98,5\%) de exames. Apenas duas equipes $(2,89 \%)$ referiram não ter disponível 
o conjunto de exames pesquisados. Quanto aos pedidos de ultrassonografia no pré-natal, $96,5 \%$ receberam resposta de agendamento.

\section{Discussão}

Embora algumas equipes não tenham aderido ao PMAQ-AB em seu primeiro ciclo, foi possível avaliar a dimensão estrutura de todas as 27 unidades de saúde da família em funcionamento no momento da adesão ao programa.

Mais de um terço dos profissionais de nível superior compunha a equipe há menos de um ano, o que pode ser reflexo da alta rotatividade desses trabalhadores. Os dados nacionais apontaram resultados pouco melhores para o tempo de trabalho na equipe, ou seja, $27,6 \%$ dos profissionais estavam atuando há menos de um ano na equipe (SEIDL ET AL., 2014). Esta pesquisa demonstra a persistência da dificuldade de fixação dos profissionais de nível superior nas EqSF, mesmo nas unidades localizadas no ambiente urbano e em município com elevado IDH. Poucas unidades de saúde da família em Niterói possuíam equipe com saúde bucal, colocando o município em desvantagem ante o perfil nacional, no qual $73 \%$ das equipes possuíam esse componente (CASOTTI ET AL., 2014).

Ao se avaliar a formação dos profissionais em Niterói, a especialidade em áreas relacionadas com a saúde da família foi baixa para médicos, mediana para enfermeiros e ausente para odontólogos. No nível nacional, os resultados foram melhores: cerca de $60 \%$ dos médicos e $70 \%$ de enfermeiros e odontólogos tinham formação em áreas de saúde da família ou saúde coletiva (SEIDL ET AL., 2014). Em Niterói, chama atenção o fato de, por um lado, o profissional médico não ter formação complementar à graduação em um quarto dos casos, provavelmente decorrente da procura dos recém-formados por essa oportunidade de trabalho, e, por outro lado, a maioria possuir mais de uma qualificação.
Como a maioria das qualificações corresponde a áreas de menor interesse para a saúde da família, provavelmente esses trabalhadores estão investindo em outras carreiras.

Em trabalhos que avaliaram estrutura das unidades especificamente para atenção pré-natal, poucos abrangeram o item de formação, relatando mais o quantitativo e distribuição dos profissionais: Rocha et al. (2012) identificaram a formação dos pré-natalistas em ginecologia-obstetrícia ou saúde da família; Oliveira et al. (2013) observaram que nas unidades básicas de saúde a formação principal dos pré-natalistas era ginecologia-obstetrícia, enquanto nas unidades de saúde da família predominavam clínica médica e saúde da família. Encontraram-se resultados parecidos, mas pediatria foi uma especialidade marcante entre os médicos de Niterói.

Os resultados sobre apoio matricial e institucional nesta pesquisa foram excelentes. Nos municípios brasileiros com aporte populacional semelhante, a maioria das EqSF relatou: apoio matricial de outros profissionais (FAUSTO ET AL., 2014); ações de educação permanente ofertadas pela gestão municipal (SEIDL ET AL., 2014) e apoio da gestão no planejamento e organização do processo de trabalho (CRUZ ET AL., 2014). O apoio matricial tem ajudado a melhorar a qualidade da $\mathrm{AB}$ no Brasil, e elevados graus de apoio matricial corresponderam a chances elevadas de as equipes obterem uma melhor certificação na atenção à mulher pelo PMAQ-AB (FONSECA SOBRINHO ET AL., 2014).

Nenhum entrevistado referiu que sua equipe possuía população cadastrada superior a 2.700 pessoas, e a média por equipe foi de 1.863 indivíduos. No âmbito nacional, um terço das equipes tinha até 4 mil pessoas sob sua responsabilidade (ALVES ET AL., 2014; TEIXEIRA ET AL., 2014). O território foi incorporado na avaliação da planta física para contemplar o sentido de responsabilidade sanitária, já que as EqSF devem ter limite territorial e populacional e manter gerência sobre estes. Praticamente todos os respondentes de 
Niterói referiam mapeamento do território, porém, menos da metade apresentou o mapa com as microáreas e os grupos prioritários sinalizados. No Brasil, os valores foram superiores aos de Niterói, mas a sinalização dos grupos de risco prioritários também foi problemática (TEIXEIRA ET AL., 2014). É possível que as equipes disponham de outras formas de gerenciamento das gestantes cadastradas como, por exemplo, espelho do cartão de gestante, porém esse tipo de gerenciamento não foi investigado nesse ciclo do PMAQ-AB.

A acessibilidade para usuários com deficiência ou dificuldade de locomoção necessita de maiores investimentos nas unidades, já que foram relatadas frequências baixas em relação aos itens pesquisados. A acessibilidade foi estudada em Pernambuco (ALBUQUeRQue ET AL., 2014), com dados do PMAQ-AB, e foi considerada igualmente incipiente.

As principais deficiências na área física das unidades de saúde se relacionaram com a ausência de sala para estoque de medicamentos, consultório odontológico, sala de acolhimento e banheiro para usuária feminina. Sobre o consultório odontológico, é possível concluir que há necessidade de investimentos nesse ambiente para agregação nas equipes dos profissionais de saúde bucal. Apesar de não haver sala para atividades educativas em $26 \%$ das unidades, todos os profissionais relataram a oferta dessas atividades, provavelmente, em outros ambientes da unidade ou mesmo fora dela, em espaços nas comunidades. Em Fortaleza, além da ausência de salas para atividades em grupo, não havia salas de espera; as usuárias ficavam nos corredores em cadeiras ou bancos inadequados para gestantes, muitas vezes em número insuficiente ou quebrados (ROCHA ET AL., 2012). Não foram avaliados no PMAQ-AB a qualidade dessas acomodações.

Os dispositivos de tecnologia da informática são primordiais para o acesso à capacitação profissional à distância, mas também para organização dos serviços e comunicação. A indisponibilidade ou inadequação desses recursos pode comprometer a alimentação adequada do Sisprenatal ou de outros bancos de dados de interesse da atenção, e inviabiliza a integração oportuna à rede. Para esses equipamentos, a maioria das unidades dispunha de computadores, mas pouco mais da metade tinha acesso à internet. O prontuário eletrônico ainda não está disponível nas unidades, e não há computadores nos consultórios.

Em Niterói, não foram detectados problemas com a disponibilidade de equipamentos e materiais, porém não foi investigada na entrevista a rotina de manutenção e calibração, apenas se estavam em condições de uso. Nos trabalhos específicos sobre estrutura do pré-natal, também não foi encontrada insuficiência de equipamentos e materiais para semiotécnica da gravidez, porém não houve uniformidade entre os itens pesquisados (XIMENES ET AL., 2008; ROCHA ET AL., 2012; OLIVEIRA ET AL., 2013; SILVA ET AL., 2013; OLIVEIRA 2014).

$\mathrm{Na}$ avaliação sobre os impressos relativos ao atendimento pré-natal, as carências detectadas foram de pequena monta e concentradas em uma unidade. Uma exceção foi justamente a caderneta da gestante, ausente em cerca de $20 \%$ das unidades de saúde. Ela é um instrumento importante para o acompanhamento pré-natal, uma vez que contém as informações primordiais sobre a gestação, tanto para a usuária como para o profissional que realizará o atendimento. Na maioria dos trabalhos publicados, os impressos foram avaliados como adequados, embora também não houvesse uniformidade entre os componentes pesquisados (OLIVEIRA ET AL., 2013; SILVA ET AL., 2013; OLIVEIRA, 2014).

Com relação aos imunobiológicos, todas as unidades dispunham das vacinas DTadulto (vacina para tétano e difteria) e contra Hepatite B; e apenas uma unidade referiu que dispunha de forma insuficiente. É relevante mencionar que foi uma das duas unidades desabastecidas de medicamentos, o que reforça a possibilidade de questões inerentes a essa unidade em especial. Vale ressaltar que a correta imunização contra o tétano é um dos 
requisitos de qualidade da atenção pré-natal, previsto pelo Programa de Humanização do Pré-Natal e Nascimento (PHPN), e que, no Brasil, a cobertura costuma ser melhor no pré-natal dos serviços públicos, comparados aos privados (RIBEIRO ET AL., 2004; CESAR ET AL., 2012).

A possibilidade de realização de exame preventivo do câncer de colo de útero e mama durante a gestação está contemplada na última publicação do Ministério da Saúde para pré-natal de baixo risco (BRASIL, 2013). Em Niterói, todos os insumos estavam disponíveis de forma adequada para a realização do colpocitológico, com exceção do fixador de lâmina. Tomasi et al. (2015) estudaram a estrutura para realização desse exame no Brasil com os dados do PMAQ-AB e perceberam que a adequação mantinha relação com o maior aporte populacional e IDH do município; era melhor em unidades de saúde da família comparadas com outras modalidades de AB; com EqSF completas e com população adscrita de até $4 \mathrm{mil}$ pessoas. Nacionalmente, a maioria das unidades possuía os insumos de forma satisfatória para o exame (TOMASI ET AL., 2015).

Os medicamentos para as unidades de saúde da família são distribuídos no município a partir de uma farmácia central. Dos 13 itens considerados pertinentes ao pré-natal e presentes no questionário do PMAQ-AB, apenas duas unidades não dispunham de nenhum deles. Estas unidades também estavam desabastecidas de outros insumos, provavelmente resultado de questões de gestão delas, uma vez que nenhuma outra apresentou esse padrão. Destaca-se como relevante a falta de penicilina benzatina em algumas unidades, dificultando o tratamento adequado da sífilis na gestante e, consequentemente, a prevenção da sífilis congênita. Nas demais, foram relatados problemas pontuais com diferentes medicamentos. Em municípios de mesmo porte populacional, três quartos das unidades de saúde da família recebiam medicamentos suficientes da farmácia básica (FAUSTO ET AL., 2014), e os grupos de medicamentos com maior disponibilidade foram os antianêmicos e analgésicos (MENDES ET AL., 2014). Em todos os trabalhos sobre estrutura do pré-natal, houve avaliação da disponibilidade de medicamentos, embora com conjuntos diferentes de fármacos, e foram apontados problemas de desabastecimento (XIMENES ET AL., 2008; SILVEIRA ET AL., 2001; ROCHA ET AL., 2012; OLIVEIRA ET AL., 2013; OLIVEIRA, 2014).

Sobre o uso de protocolos para a efetivação da coordenação do cuidado clínico, a maior parte das equipes de Niterói e do Brasil apresentou o dispositivo para o pré-natal (FAUSTO ET AL., 2014). Em Botucatu-SP, o protocolo foi mais encontrado nas unidades de saúde da família que nas unidades básicas, explicado pela maior presença de enfermeiros como prénatalistas (OLIVEIRA ET AL., 2013).

As visitas domiciliares durante a gestação e no pós-parto são atividades inerentes à saúde da família. Em todas as entrevistas do PMAQ-AB em Niterói, os profissionais referiram organização de agenda para visitas domiciliares, assim como a maior parte das equipes, na avaliação nacional, tinha essa organização (ALVES ET AL., 2014). Contudo, na maioria das respostas, as equipes do município de Niterói não conheciam critérios para priorização dessas visitas, o que pode comprometer a realização oportuna destas para gestantes e puérperas.

Em Niterói, 92,7\% das equipes tinham agendas organizadas para realização de grupos de educação em saúde para gestantes e puérperas, comparado a percentuais um pouco inferiores na região Sudeste e no País (TEIXEIRA ET AL., 2014). Nesse município, todas as 27 unidades de saúde pesquisadas ofereciam o serviço à população.

A avaliação da disponibilidade de apoio laboratorial foi prejudicada pela ausência de questões relativas a vários exames: tipo sanguíneo e fator Rh, teste de Coombs, teste oral de tolerância à glicose, urocultura com antibiograma, bacterioscopia do conteúdo vaginal e eletroforese de proteínas. Os exames laboratoriais que puderam ser pesquisados estavam disponíveis para a quase 
totalidade das equipes. Boa cobertura laboratorial também foi relatada por Rocha et al. (2012) e Oliveira (2014) em seus trabalhos. No entanto, registraram-se problemas com o recebimento dos resultados dos exames por algumas equipes. Em Niterói, não foram detectados problemas para agendamento de exame de ultrassonografia obstétrica.

A definição das diretrizes dos fluxos e contrafluxos de atendimento e o mapeamento da rede de serviços de saúde, por gestores e profissionais da saúde, são importantes, pois estabelecem a base para o sistema de referência para população (PROTASIO ET AL., 2014). Quanto maior o porte populacional das cidades, mais alto foi o percentual de respostas positivas para essas questões (ALVES ET AL., 2014). Entre as EqSF entrevistadas no País e em Niterói, a quase totalidade declarou haver uma central de regulação, uma central de marcação de consultas especializadas e de exames. No entanto, em relação ao ordenamento de fluxos assistenciais, 72,5\% das equipes de Niterói e menos da metade no Brasil (ALVES ET AL., 2014) referiram dispor de protocolos para a priorização de casos mais complexos. Especificamente em relação às gestantes, $13 \%$ das equipes em Niterói informaram não possuir maternidade definida para as gestantes acompanhadas, resultado semelhante aos relatados em nível nacional (ALVES ET AL., 2014), contrariando as orientações da Rede Cegonha.

Em relação às características gerais das unidades, o horário de funcionamento era semelhante à maioria das unidades de saúde em nível nacional. Esse horário, restrito ao período diurno, é considerado fator impeditivo para a população trabalhadora chegar aos serviços de saúde, diminuindo a 'acessibilidade temporal' (ESCOREL ET AL., 2007; FAUSTO ET AL., 2014; ALVES ET AL., 2014), sendo desejável horário de atendimento expandido até a noite e em finais de semana.

Entre as diretrizes do PMAQ-AB, está o estímulo ao 'processo contínuo de melhoramento dos indicadores de acesso e de qualidade'. Nesse contexto, os resultados deste trabalho contribuíram para detectar os pontos fortes e as fragilidades da estrutura das unidades de saúde quanto ao pré-natal, em Niterói. Com base nesses resultados, os gestores podem direcionar esforços para melhorar as condições de acessibilidade, qualificar os profissionais, estimular a permanência e o vínculo, ampliar a capacitação das equipes quanto ao gerenciamento do território e dos protocolos de risco.

Cabe reforçar a importância da caderneta da gestante como instrumento de registro, acompanhamento e facilitador dos fluxos de informação no atendimento à mulher. A questão de maternidade de referência extrapola ações isoladas da AB e deve ser contemplada no componente relativo à atenção ao parto, no âmbito da Rede Cegonha.

De acordo com o preconizado pelo Ministério da Saúde (BRASIL, 2013), os resultados encontrados sugerem que o município de Niterói oferece, em suas unidades da ESF, condições de privacidade e parte dos equipamentos, medicamentos e exames para o atendimento pré-natal. Quanto à ambiência, é necessário melhorar condições de ventilação, limpeza e acústica; no que concerne à estrutura predial, deve-se valorizar os espaços destinados a acolhimento e prover sala adequada para estoque de medicamentos. A dimensão de pior adequação foi a acessibilidade, que deve receber maior atenção por parte dos gestores.

\section{Considerações finais}

Este trabalho buscou abranger o máximo de aspectos relacionados com a dimensão estrutura da assistência pré-natal. Optou-se por não usar classificações quantitativas da adequação, considerando que não há trabalhos com dados do PMAQ-AB que avaliem estrutura para atendimento ao pré-natal, e que a discussão neste momento deveria priorizar a dimensão qualitativa. Todavia, fica a sugestão 
de trabalhos que desenvolvam índices de avaliação de estrutura das unidades de saúde para o atendimento do pré-natal, uma vez que esse índice ainda não existe.

Sobre o banco de dados utilizado, o PMAQ-AB mostrou-se uma ferramenta útil para avaliar a maioria dos itens relativos à estrutura do pré-natal, mas precisa de aperfeiçoamentos. Por ter sido desenvolvido para avaliação da $\mathrm{AB}$ como um todo, é compreensível que não contemple todos os detalhes considerados importantes, mas foi possível extrair um conjunto extenso de elementos para a análise da dimensão estrutura. Dessa forma, cabe sugerir algumas inclusões como os exames mínimos recomendados pelo Ministério da Saúde (BRASIL, 2013) para o pré-natal de baixo risco. Por outro lado, com esse instrumento, é possível uniformizar as avaliações nacionais no conjunto de quesitos e tempo de realização da pesquisa. Como proposta do próprio PMAQ-AB, também será possível acompanhar na linha do tempo a adequação das unidades pesquisadas e perceber os investimentos realizados pela gestão e qualificação dos respondentes.

Para o município de Niterói, identificou-se adequação entre regular e boa para a estrutura dos serviços de pré-natal. Com os resultados desta avaliação, é possível sugerir incentivos na qualificação pessoal dos recursos humanos em áreas de interesse para a saúde da família. Tem sido ampliada a possibilidade de formação dos profissionais pelo Ministério da Saúde, e isto deve se refletir, futuramente, na qualificação dos profissionais lotados nas unidades, se houver também incentivos por parte da gestão local. Melhorias na tecnologia da informática podem facilitar e dinamizar algumas tarefas gerenciais das equipes, melhorar a alimentação dos sistemas de informação da $\mathrm{AB}$ e resolver o problema da demora no recebimento dos resultados dos exames, detectado em algumas equipes.

Sugere-se também acompanhamento da apropriação por parte das equipes dos dados do território de abrangência, como ferramenta para melhoria da assistência, pelo monitoramento do território e da população. Recomenda-se a investigação dos determinantes da não fixação dos profissionais de nível superior nas equipes de trabalho, o que influencia negativamente na longitudinalidade do cuidado, nas relações das equipes com as populações assistidas e na aderência dos usuários aos projetos terapêuticos. Deve ser garantida a disponibilidade da caderneta da gestante em todas as unidades e maternidade de referência para todas as parturientes.

Cabe ressaltar que é de extrema importância a adequação dos insumos necessários para o diagnóstico e tratamento oportuno das doenças sexualmente transmissíveis nas unidades, especialmente em gestantes, para evitar a transmissão vertical. Os resultados encontrados também apontam para a necessidade de aprimoramentos em relação a alguns aspectos da ambiência e da estrutura predial e, destacadamente, à acessibilidade das unidades, além da incorporação do consultório odontológico.

Os resultados positivos principais foram com relação ao apoio matricial e institucional, à presença da maioria dos materiais e insumos e ao sistema de regulação, que devem ser preservados pelo gestor local e incrementados sempre que necessário.

Outros estudos sobre a atenção pré-natal em Niterói que avaliem os demais componentes da qualidade dos serviços - processo e resultados - devem ser desenvolvidos, assim como outras pesquisas sobre a estrutura, a partir das subsequentes avaliações externas do PMAQ-AB para observação das adequações ao longo do tempo.

\section{Colaboradores}

As três autoras contribuíram substancialmente para concepção, análise e interpretação dos dados, redigiram em conjunto o manuscrito e participaram da aprovação da versão final. 


\section{Referências}

ALBUQUERQUE, M. S. V. Acessibilidade aos serviços de saúde: uma análise a partir da atenção básica em Pernambuco. Saúde debate, Rio de Janeiro, v. 38, n. esp, p. 182-94, out. 2014.

ALVES, M. G. M. et al. Fatores condicionantes para o acesso às equipes da Estratégia Saúde da Família no Brasil. Saúde debate, Rio de Janeiro, v. 38, n. esp., p. 34-51, out. 2014

BRASIL. Ministério da Saúde. Instrumento de avaliação externa da saúde mais perto de você - acesso e qualidade: Programa Nacional de Melhoria do Acesso e da Qualidade da Atenção Básica (PMAQ). Brasília, DF, 201la. Disponível em: <http://dab.saude.gov.br/ portaldab/ape_pmaq.php>. Acesso em: 22 nov. 2016.

Ministério da Saúde. Portaria n ${ }^{\circ} 1.654$, de 19 de julho de 2011. Institui, no âmbito do Sistema Único de Saúde, o Programa Nacional de Melhoria do Acesso e da Qualidade da Atenção Básica (PMAQ-AB) e o Incentivo Financeiro do PMAQ-AB, denominado Componente de Qualidade do Piso de Atenção Básica Variável - PAB Variável. Diário Oficial [da] União. Brasília, DF, 20 jul. 2011b. Disponível em: <http://www. sgc.goias.gov.br/upload/links/arq_416_portariaPMAQ. pdf>. Acesso em: 22 nov. 2016.

Ministério da Saúde. Secretaria de Atenção à Saúde. Departamento de Atenção Básica. Atenção ao pré-natal de baixo risco. Brasília, DF: Ministério da Saúde: 2013. (Caderno de atenção básica, 32). Disponível em: <http://dab.saude.gov.br/portaldab/ biblioteca.php? conteudo=publicacoes $/$ cab32 $>$. Acesso em: 22 nov. 2016.

CASOTTI, E. et al. Atenção em saúde bucal no Brasil: uma análise a partir da avaliação externa do PMAQ-AB. Saúde debate, Rio de Janeiro, v. 38, n. esp., p. 140-57, out. 2014.

CESAR, J. A. et al. Assistência pré-natal nos serviços públicos e privados de saúde: estudo transversal de base populacional em Rio Grande, Rio Grande do Sul, Brasil. Caderno de Saúde Pública, Rio de Janeiro, v. 28, n. 11, p. 2106-2114, nov. 2012.

CRUZ, M. M. et al. Usos do planejamento e auto avaliação nos processos de trabalho das equipes de saúde da família na atenção básica. Saúde debate, Rio de Janeiro, v. 38, n. esp., p. 124-139, out. 2014

ESCOREL, S. et al. O Programa de Saúde da Família e a construção de um novo modelo para a Atenção Básica no Brasil. Revista Panamericana de Salud Publica, Washington, v. 21, n. 2-3, p. 164-176, fev./mar. 2007.

FAUSTO, M. C. R. et al. A posição da Estratégia Saúde da Família na rede de atenção à saúde na perspectiva das equipes e usuários participantes do PMAQ-AB. Saúde debate, Rio de Janeiro, v. 38, n. esp., p. 13-33, out. 2014.

FONSECA, C. R. B. et al. Adequacy of antenatal care and its relationship with low birth weight in Botucatu, São Paulo, Brazil: a case-control study. BMC Pregnancy Childbirth, London, n. 14, p. 255, ago. 2014.

FONSECA SOBRINHO, D. et al. Compreendendo o apoio matricial e o resultado da certificação de qualidade nas áreas de atenção à criança, mulher, diabetes/hipertensão e saúde mental. Saúde debate, Rio de Janeiro, v. 38, n. esp., p. 83-93, out. 2014.

GARNELO, L. et al. Organização do cuidado às condições crônicas por equipes de saúde da família na Amazônia. Saúde debate, Rio de Janeiro, v. 38, n. esp., p. 158-72, out. 2014.

\section{INSTITUTO BRASILEIRO DE GEOGRAFIA E ESTATÍSTICA (IBGE). IBGE/Cidades/Rio de Janeiro/ Niterói. Disponível em: <http://cidades.ibge.gov.br/ painel/painel.php?codmun=330330 $>$. Acesso em: 12 mar. 2016.}

MENDES L V. et al. Disponibilidade de medicamentos 
nas unidades básicas de saúde e fatores relacionados: uma abordagem transversal. Saúde debate, Rio de Janeiro, v. 38, n. esp., p. 109-23, out. 2014.

OLIVEIRA, R. L. A. et al. Avaliação da atenção prénatal na perspectiva dos diferentes modelos na atenção primária. Revista Latino Americana de Enfermagem, São Paulo, v. 21, n. 2, p. 546-553, mar./abr., 2013.

OLIVEIRA, C. R. T. Avaliação da qualidade da atenção pré-natal em 13 unidades da estratégia saúde da família no município de Juazeiro do Norte (CE). 2014. 106 f. Dissertação (Mestrado Profissional em Saúde da Família) - Universidade Federal do Ceará, Fortaleza, 2014.

PROTASIO, A. P. L. et al. Avaliação do sistema de referência e contra referência do estado da Paraíba segundo os profissionais da atenção básica no contexto do $1^{\circ}$ ciclo de avaliação externa do PMAQ-AB. Saúde debate, Rio de Janeiro, v. 38, n. esp., p. 209-220, out. 2014.

RIBEIRO, J. M. et al. Atenção ao pré-natal na percepção das usuárias do Sistema Único de Saúde: um estudo comparativo. Cad. Saúde Pública, Rio de Janeiro, n. 20 , p. $534-545$, abr. 2012.

ROCHA, R. S.; SILVA, M. G. C. Assistência prénatal na rede básica de Fortaleza-CE: uma avaliação da estratégia, do processo e do resultado. Revista Brasileira de Promoção à Saúde, Fortaleza, v. 2, n. 3, p. 344-355, jul./ago., 2012.
SEIDL, H. V. S. P. et al. Gestão do trabalho na atenção básica em saúde: uma análise a partir da perspectiva das equipes participantes do PMAQ-AB. Saúde debate, Rio de Janeiro, v. 38, n. esp., p. 94-108, out. 2014.

SILVA, E. P. et al. Pré-natal na atenção primária do município de João Pessoa-PB: caracterização de serviços e usuárias. Rev. Bras. de Saúde Matern. Infant., Recife, v. 13, n. 1, p. 29-37, jan./mar. 2013.

TEIXEIRA, M. B. et al. Avaliação das práticas de promoção da saúde: um olhar das equipes participantes do Programa Nacional de Melhoria do Acesso e da Qualidade da Atenção Básica. Saúde debate, Rio de Janeiro, v. 38, n. esp., p. 52-68, out. 2014.

TOMASI, E. et al. Estrutura e processo de trabalho na prevenção do câncer de colo de útero na atenção básica à saúde no Brasil: Programa de Melhoria do Acesso e da Qualidade - PMAQ. Rev. Bras. de Saúde Matern. Infant., Recife, v. 15, n. 2, p. 171-180, abr./jun. 2015.

XIMENES NETO, F. R. G. et al. Qualidade da atenção ao pré-natal na estratégia de saúde da família em Sobral, Ceará. Rev. bras. enferm., Brasília, DF, v. 61, n. 5, p. 595-602, set./out. 2008 .

\footnotetext{
Recebido para publicação em agosto de 2016

Versão final em novembro de 2016

Conflito de interesses: inexistente

Suporte financeiro: não houve
} 